\title{
IncRNA expression character associated with ischemic reperfusion injury
}

\author{
XIAOWEI WU*, HONGYI ZHU*, SUHUA ZHU, MAOJUAN HAO and QINGPING LI \\ Department of Pharmacology, School of Basic Medical Sciences, Nanjing Medical University, Nanjing, \\ Jiangsu 210029, P.R. China
}

Received May 11, 2016; Accepted March 30, 2017

DOI: $10.3892 / \mathrm{mmr} .2017 .7051$

\begin{abstract}
Ischemic reperfusion injury (IRI) contributes to morbidity and mortality worldwide and results in a poor outcome for patients suffering from myocardial infarction. Ischemic post-conditioning (IPostC), consisting of one or several brief periods of ischemia and reperfusion, generates powerful protection against IRI. The mechanism of IPostC initiation and development has previously been investigated, however still remains to be fully elucidated. Notably, long non-coding (lnc) RNAs have previously been demonstrated to be important in cardiovascular diseases. However, there is little information about the systematic analysis of IRI-associated lncRNA expression signature. The present study used microarrays to analyze the lncRNA expression characters of ischemic IPostc (corresponding to IRI), and demonstrated that 2,292 lncRNAs were observed to be upregulated and 1,848 lncRNAs downregulated. Gene ontology (GO) and Pathway analysis subsequently demonstrated that dysregulated lncRNAs participated in various biological processes, which are upregulated or downregulated in IPostC tissues. Finally, the present study verified that AK144818, ENSMUST00000156637, ENS MUST00000118342, ENSMUST00000118149, uc008ane.1, ENSMUST00000164933, ENSMUST00000162347, ENS MUST00000135945, and ENSMUST00000176338, ENS MUST00000120587, ENDMUST00000155271, ENSMUS T00000125121 and Uc008thl.1 were associated with the initiation and development of IPostC. The present study may aid in the understanding of the initiation and development mechanisms of IPostC and provide novel and potential biomarkers that may be used in the diagnosis or as therapeutic targets in the treatment of IRI.
\end{abstract}

Correspondence to: Dr Qingping Li, Department of Pharmacology, School of Basic Medical Sciences, Nanjing Medical University, 140 Hanzhong Road, Nanjing, Jiangsu 210029, P.R. China

E-mail: qpli@njmu.edu.cn

${ }^{*}$ Contributed equally

Key words: ischemic reperfusion injury, ischemic post-conditioning, long non-coding RNA

\section{Introduction}

Ischemic heart disease is a leading cause of morbidity worldwide (1). Coronary thrombosis induces ischemic heart disease, and rapid reperfusion of the ischemic heart is currently the primary therapeutic strategy to improve the survival outcome of patients. However, restoration of cardiac circulation is accompanied by cell damage and death, reperfusion arrhythmias, myocardial stunning and vascular defects with the no-reflow phenomena (2), which are involved in ischemic reperfusion injury (IRI).

The mechanism underlying IRI initiation has previously been studied, and it has been demonstrated that causes of IRI include calcium overload, oxidative stress, mitochondrial dysfunction and activation of apoptotic and autophagy pathways in ischemic reperfusion. Alterations in intracellular calcium $\left[\mathrm{Ca}^{2+}\right]$ i regulation, including $\mathrm{Ca}^{2+}$ release from the sarcoplasmic reticulum, have been demonstrated to be included in the cytosolic $\mathrm{Ca}^{2+}$ overload and cardiac dysfunction during IRI and heart failure (3-5). Recent studies suggest that these mechanisms are interrelated and reactive oxygen species (ROS) overproduction may increase abnormal $\mathrm{Ca}^{2+}$ regulation by depressing sarcoplasmic reticulum $\mathrm{Ca}^{2+}$ uptake and release activities $(6,7)$. In turn, increased cytosol calcium concentrations may induce $\operatorname{ROS}$ production $(8,9)$. Furthermore, oxidative stress, $\mathrm{Ca}^{2+}$ overload, decreased ATP levels and increased matrix $\mathrm{pH}$ induce the formation of a large number of mitochondrial permeability transition pores (mPTP) in the mitochondrial membrane. The mPTP openings may result in matrix swelling, depolarization of the mitochondrial membrane potential, decrease of ATP synthesis and the release or activation of various pro-apoptotic proteins, including cytochrome $c(10)$. These processes rapidly lead to irreversible damage to the mitochondria and further necrosis and apoptosis of cardiac myocytes $(11,12)$.

In order to reduce the damage resulting from IRI, several therapeutic strategies have been clinically performed, including ischemic pre-conditioning (IPC) and ischemic post-conditioning (IPostC). However, the attendant risks accompanying repeated clamping and declamping of the aorta have limited the clinical application of IPC. IPostC consists of one or several brief periods of ischemia and reperfusion and generates protection against IRI (13). The IPostC protocol was previously demonstrated to reduce myocardial injury 
via a decrease in peri-operative troponin- $T$ and creatine kinase-myocardial $b$ fraction release and fewer inotrope requirements post-surgery. Therefore, in order to improve the survival outcome of myocardial infarction patients, the mechanism of IRI requires further comprehensive studies which may lead to the identification of potential therapeutic targets.

Long non-coding RNAs (lncRNAs) are a group of RNAs which are $>200$ nucleotides in length, with little translation capacity, and the total quantity may reach $\sim 410,000$ (14-17). The primary functions of lncRNAs include regulation of gene methylation, transcriptional activation and conjugation with mRNAs and miRNAs to affect translation progression (18-20). lncRNAs exhibit sense overlapping, anti-sense, intronic, divergent and intergenic associations with their adjacent coding genes $(21,22)$. Aberrant expression level of lncRNAs is known to be associated with various malignant biological processes including carcinogenesis, cell proliferation, apoptosis, migration, invasion and autophagy (23-26). Furthermore, Archer et al (25) reported that lncRNAs may be used as novel therapeutics in cardiovascular medicine. However, information and specific mechanisms regarding the differentially expressed lncRNAs between IRI and IPostC tissues remain to be elucidated. The present study aimed to identify the dysregulated lncRNAs present in IPostC (compared with IRI), which may help to understand the initiation and development mechanism underlying IPostC and determine any potential biomarkers which may be of value in the future diagnosis or therapeutic treatment of IRI.

\section{Materials and methods}

Animals and RNA extraction. All animal experimental procedures were performed in accordance with the National Institutes of Health Guide for the Care and Use of Laboratory Animals and were approved by the Institutional Ethics Committee of Nanjing Medical University (Nanjing, China). All experiments were performed with age-matched male C57BL6 mice (8-10 weeks; 24-28 g) were obtained from Model Animal Institute of Nanjing University (Nanjing, China). The mice were maintained at $22^{\circ} \mathrm{C}$, humidity of $50 \%$ with 12 -h light/dark cycle. A total of 10 mice were used for the present study and the treatment period of the model was $170 \mathrm{~min}$. At $12 \mathrm{~h}$ prior to the experiment, the mice were without food and water (27).

The Langendorff preparation (Radnoti LLC, Monovia, CA, USA) was performed as previously described (26). Briefly, following anesthetization with chloralic hydras $(15 \mathrm{ml} / \mathrm{kg})$, the mouse hearts were isolated. The ascending aorta was cannulated with a blunt needle. The heart was perfused at a constant pressure of $100 \mathrm{~cm} \mathrm{H}_{2} \mathrm{O}$ with modified Krebs-Henseleit buffer (in mM: $118 \mathrm{NaCl}, 4.7 \mathrm{KCl}, 1.2 \mathrm{MgSO}_{4}, 1.2 \mathrm{KH}_{2} \mathrm{PO}_{4}$, $1.8 \mathrm{CaCl}_{2}, 25 \mathrm{NaHCO}_{3}$ and 11 glucose), which was maintained at $37^{\circ} \mathrm{C}$ and bubbled continuously with a mixture of $95 \%$ $\mathrm{O}_{2}$ and $5 \% \mathrm{CO}_{2}$. Global ischemia was induced by cessation of perfusion, followed by reperfusion. Isolated hearts from wild-type mice were exposed to IRI and IPostC. IRI samples underwent $20 \mathrm{~min}$ equilibration, $30 \mathrm{~min}$ ischemia and $120 \mathrm{~min}$ reperfusion. The IPostC samples were exposed to $20 \mathrm{~min}$ equilibration, $30 \mathrm{~min}$ ischemia followed by $10 \mathrm{sec}$ reperfusion and $10 \mathrm{sec}$ ischemia, over 3 cycles (Fig. 1). All the samples were immediately frozen in liquid nitrogen and stored at $-80^{\circ} \mathrm{C}$ prior to analysis. A total of two animal samples were randomly selected for mouse lncRNA microarray analysis. Total RNA was extracted from 2 IRI samples and 3 IPostC samples using TRIzol ${ }^{\circledR}$ reagent (Invitrogen; Thermo Fisher Scientific, Inc., Waltham, MA, USA). The samples were quantified and quality assessed using Nanodrop and Agilent 2100 Bioanalyzer (Agilent Technologies, Inc., Santa Clara, CA, USA), respectively. RNA quantity was measured by NanoDropND-1000.

Assessment of myocardial infarct size (IS). IS was determined as previously described (26). At the end of experiments, mouse hearts were perfused with triphenyltetrazolium chloride for $1 \mathrm{~min}$ and then incubated with $1 \%$ triphenyltetrazolium chloride at $37^{\circ} \mathrm{C}$ for $15 \mathrm{~min}$. Following freezing at $-80^{\circ} \mathrm{C}$, hearts were transected into 5 sections with thickness of $2 \mathrm{~mm}$ (28). Cardiac sections were incubated with $10 \%$ formalin for $30 \mathrm{~min}$. All sides of each section were photographed, as represented in Fig. 2. Viable myocardium stained red and infarcted tissue was white. The areas of red and white color in the left ventricle (LV) and the muscle area of cardiac sections were measured by computerized planimetry (ImageJ; National Institutes of Health, Bethesda, MD, USA). Total LV infarct weight was determined using the following equation: $\left[\left(\mathrm{S}_{1} / \mathrm{C}_{1}\right.\right.$ $\left.\mathrm{x} \mathrm{W}_{1}\right)+\left(\mathrm{S}_{2} / \mathrm{C}_{2} \mathrm{x} \mathrm{W}_{1}\right)+\left(\mathrm{S}_{2} / \mathrm{C}_{2} \mathrm{x} \mathrm{W}_{2}\right)+\left(\mathrm{S}_{3} / \mathrm{C}_{3} \mathrm{x} \mathrm{W}\right)+\left(\mathrm{S}_{4} / \mathrm{C}_{4}\right.$ $\left.\mathrm{x} \mathrm{W}_{4}\right)+\left(\mathrm{S}_{5} / \mathrm{C}_{5} \times \mathrm{W}_{5}\right)$ ], where $\mathrm{S}$ was area of $\mathrm{LV}$ infarction for the slice represented by the subscript, $\mathrm{C}$ was the muscle area of the cardiac section and $\mathrm{W}$ was the weight of that respective section. Total weight of viable myocardium in LV was calculated in a similar fashion. IS was calculated as a percentage of LV as follows: IS/LV = total infarct weight/(total infarct weight + total weight of viable myocardium) $\times 100 \%$.

Microarray analysis. Sample preparation and microarray hybridization were performed by KangChen Bio-tech Inc. (Shanghai, China). Briefly, RNA was purified from $1 \mu \mathrm{g}$ total RNA following removal of rRNA, using mRNA-ONLY Eukaryotic mRNA Isolation kit (Epicentre; Illumina, Inc., San Diego, CA, USA). Then, each sample was amplified and transcribed into fluorescent RNA along the entire length of the transcripts without bias using a random priming method as previously described (29). The labeled cRNAs were hybridized onto the Human LncRNA Array, version 2.0 (8660 K; Arraystar Inc., Rockville, MD, USA). Subsequently, the arrays were scanned by the Agilent Scanner G2505B and Agilent Feature Extraction software (version 10.7.3.1) was utilized to analyze acquired array images (Agilent Technologies, Inc.) Quantile normalization and subsequent data processing were carried out using the GeneSpring GX version 11.5.1 software package (Agilent Technologies, Inc.). Differentially expressed lncRNAs and mRNAs were identified via fold change filtering (fold change $>5.0)$, standard Student's t-test $(\mathrm{P}<0.05)$ and multiple hypothesis testing [false discovery rate (FDR) $<0.05$ ]. P-values and FDR were calculated by Microsoft Excel and MATLAB version 7.0 (MathWorks, Natick, MA, USA) respectively. The microarray data was deposited in the NCBI Gene Expression Omnibus (GEO) and the GEO accession number is GSE55191 (www.ncbi.nlm.nih.gov/geo/query/acc. cgi?acc=GSE5519 1). Pathway analysis and GO analysis were applied to determine the roles of these differentially expressed 


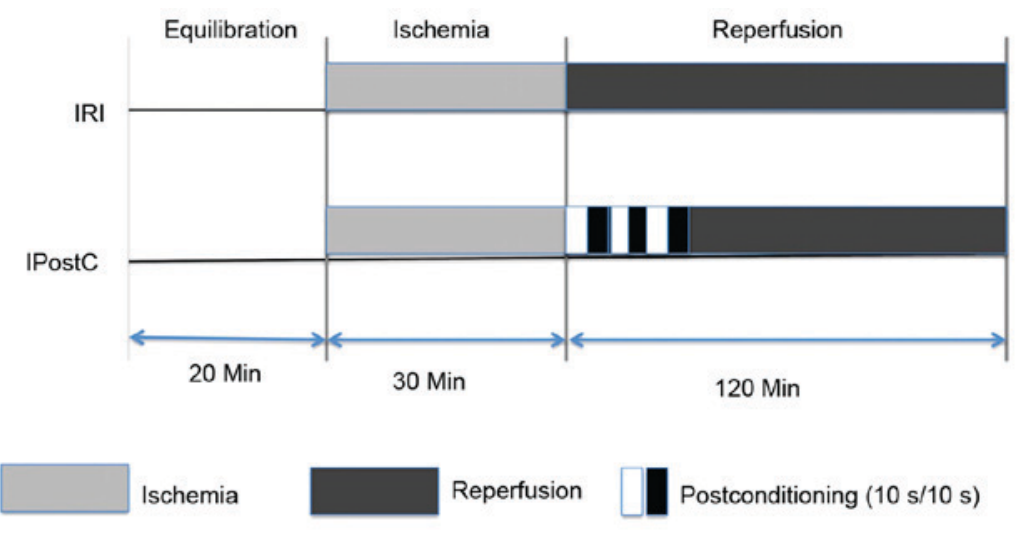

Figure 1. Illustration of the experimental protocol. All hearts were subjected to $30 \mathrm{~min}$ global ischemia followed by 120 min reperfusion. The difference between IRI and IpostC was the $10 \mathrm{sec}$ reperfusion and $10 \mathrm{sec}$ ischemia cycle repeated 3 times, followed by 30 min ischemia. IRI, ischemic reperfusion injury; IPostC, ischemic post-conditioning; s, sec; min, minutes.

A

IRI
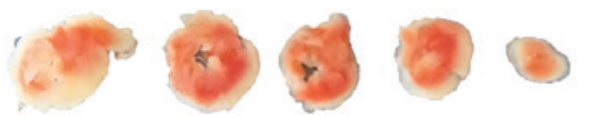

IPostC
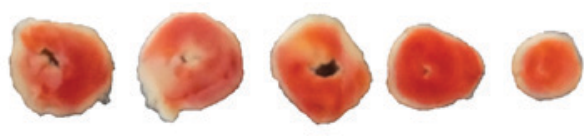

B

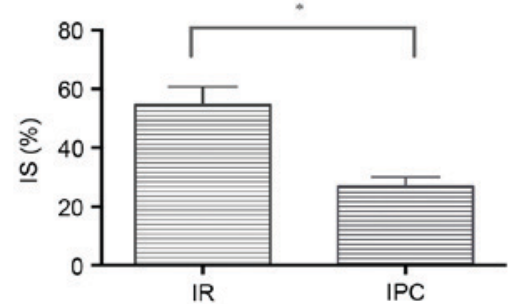

Figure 2. Myocardial IS following IRI. (A) Cardiac sections of 1 representative heart from each group. Isolated hearts from IRI and IPostC mice were cut into 5 sections and stained with triphenyltetrazolium chloride. (B) Analysis of IS with myocardial infarct area assessed by ImageJ software $\mathrm{P}<0.01 ; \mathrm{n}=6-8$ /group. IRI, ischemic reperfusion injury; IPostC, ischemic post-conditioning; IS, infarct size.

mRNAs in biological pathways or gene ontology (GO) terms. Differentially regulated mRNAs were uploaded into the Database for Annotation, Visualization and Integrated Discovery (www.david.abcc.ncifcrf.gov/) to analyze the enrichment of the coding genes, and the annotation summary results are present on the webserver.

Reverse transcription-quantitative polymerase chain reaction $(R T-q P C R)$. Total RNA of heart tissues was isolated with the RNeasy Mini kit (Qiagen, Inc., Valencia, CA, USA). A total of $1 \mu \mathrm{g}$ of RNA from each sample was reverse transcribed to cDNA using random hexamer primer with Thermo Scientific $^{\mathrm{TM}}$ RevertAid First-Strand cDNA Synthesis kit (Thermo Scientific Fisher, Inc.). Primers for each LncRNA were designed according to Primer 3 (www.sourceforge. net/projects/primer3/) online and checked with Basic Local Alignment Search Tool (BLAST, http://www.ncbi.nlm.nih. gov/BLAST/) of NCBI to ensure a unique amplification product. Quantitative PCR was performed on an Applied
Biosystems ViiA ${ }^{\mathrm{TM}} 7 \mathrm{Dx}$ (Thermo Fisher Scientific, Inc.) using the SYBR-Green (Thermo Fisher Scientific, Inc.) method according to the manufacturer's protocol. The PCR reaction conditions were as follows: a denaturation step at $95^{\circ} \mathrm{C}$ for $10 \mathrm{~min}$, followed by 40 cycles at $95^{\circ} \mathrm{C}$ for $15 \mathrm{sec}$ and 60 for $1 \mathrm{~min}$. Relative gene expression levels were quantified based on the cycle threshold $(\mathrm{Cq})$ values and normalized to the internal control gene, $\beta$-actin. All the primer sequences used were shown in Table I. The $2^{-\Delta \Delta \mathrm{Cq}}(30)$ method was used to comparatively quantify the levels of mRNA.

GO and pathway analysis. Differentially expressed lncRNAs were identified by fold-change filtering (absolute fold-change $>2.0)$, standard Student's t-test $(\mathrm{P}<0.05)$ and multiple hypothesis testing (FDR <0.05) (31). GO and pathway analysis for differentially expressed lncRNAs (antisense lncRNA, intronic lncRNA, enhancer lncRNA, and lincRNAs) were used to identify the significantly enriched biological terms and pathways. The exact function and mechanism of lncRNAs remain to be elucidated, however, previous studies have demonstrated that mammalian lncRNAs are preferentially located adjacent to genes with developmental functions. Pathway and GO analyses were applied to determine the roles of these close coding genes in biological pathways or GO terms. The GO is a controlled vocabulary composed of $>38,000$ precise defined phrases called GO terms that describe the molecular actions of gene products, the biological processes in which those actions occur and the cellular locations where they are present (32). Fisher's exact test was used to determine if there was any further overlap between the differentially expressed and the GO annotation list, than expected. The $\mathrm{p}$-value denotes the significance of GO term enrichment in the differentially expressed genes. The lower the P-value, the greater the significance of the GO term (P-value $<0.05$ is recommended). Pathway analysis is currently the primary choice for gaining insight into the underlying biology of differentially expressed genes and proteins, as it reduces complexity and has increased explanatory power (33). Pathway analysis is a functional analysis mapping genes to Kyoto Encyclopedia of Genes and Genomes pathways. The p-value (EASE-score, Fisher p-value or hypergeometric p-value) denotes the significance of the pathway 


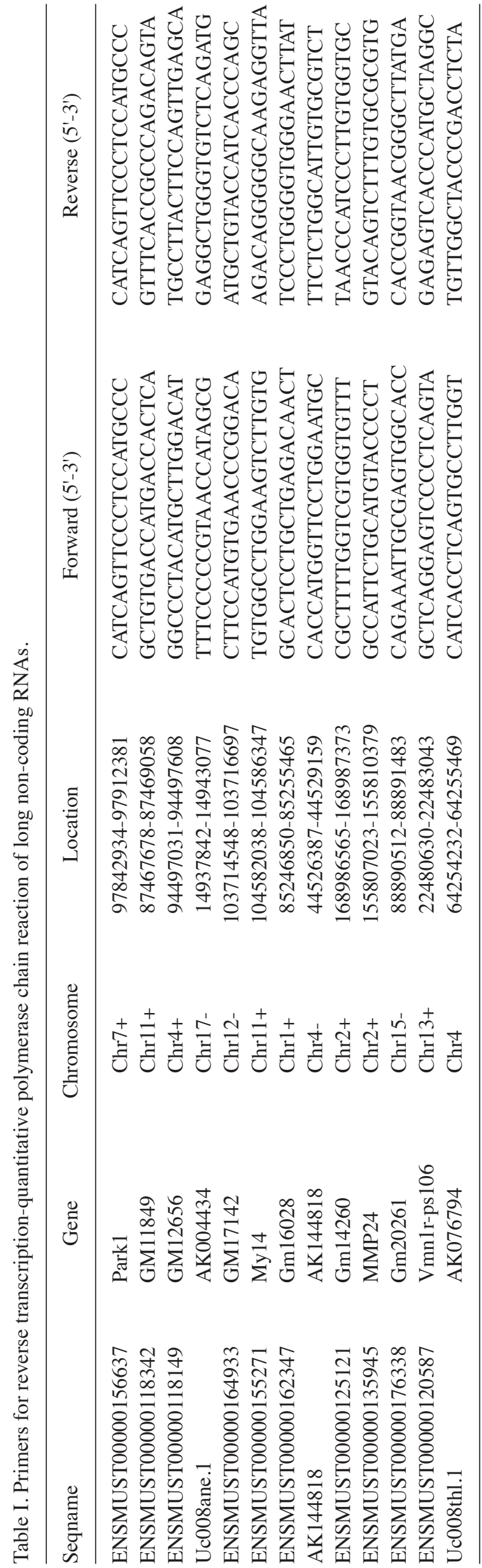

associated with the conditions. $\mathrm{P}<0.05$ was considered to indicate a statistically significant difference.

Bioinformatics analysis. By using the UCSC genome browser (www.genome.ucsc.edu/) and other databases, the sequences of lncRNAs and their associated coding genes were obtained. Numerous transcriptional regulatory elements exist in the non-coding regions, which are difficult to distinguish just using the primary sequences as a guide. The present study aimed to map various epigenetic phenomena to aid in the identification of non-coding regulatory elements. DNA methylation is essential for normal development and has been implicated in various pathological conditions (34-36). Tri-methylation of lysine 4 (H3K4me3) and Tri-methylation of lysine 27 (H3K27me3) occurs on the promoter region (37). H3K4me3 is catalyzed by trithorax-group proteins and is associated with activation, whereas $\mathrm{H} 3 \mathrm{~K} 27 \mathrm{me} 3$ is catalyzed by polycomb-group proteins and is associated with silencing $(32,38)$. The present study used the Broad H3 ChIPseq Track on the UCSC genome browser (http://genome.ucsc.edu) for mapping chromatin state.

Statistical analysis. The data were analyzed using SPSS software, version 13.0 (SPSS, Inc., Chicago, IL, USA). Differential expression levels of lncRNAs were compared using an independent-samples t-test, between two groups. Fisher's exact test was used in GO and pathway analysis. All values are expressed as the mean \pm standard deviation from at least three independent experiments. $\mathrm{P}<0.05$ was considered to indicate a statistically significant difference.

\section{Results}

Infarct size of IR and IPostC. The authors previously demonstrated that IPostC confers cardioprotection against IR injury in isolated mouse hearts (10). In the present study, IS was decreased in the IPostC samples, compared with IRI (Fig. 2), which suggested that IPostC abolishes the harmful outcome of IRI.

Differential IncRNA expression characters between IR and IPostC tissues. The present study detected the expression levels of lncRNAs in 2 IR and 3 age-matched IPostC samples via a high-throughput microarray technique. Based on the results of the microarray, 2,292 IncRNAs were observed to be upregulated and 1,848 downregulated in IPostC, compared with IRI tissue (Fig. 3A and B), with fold-change filtering (absolute fold-change $>2.0)$, standard student t-test $(\mathrm{P}<0.05)$ and multiple hypothesis testing (FDR $<0.05)$. According to the locational association of the nearby coding genes, these differentially expressed lncRNAs primarily included 446 natural antisense, 361 intronic antisense, 149 intron sense-overlapping, 1,679 intergenic, 1,356 exon sense-overlapping and 144 bidirectional lncRNAs (Fig. 3C).

\section{Discussion}

Despite being the most effective means of limiting infarct size, coronary reperfusion results in adverse effects and induces additional damage to the myocardium. IRI contributes to morbidity and mortality worldwide and is currently 
A

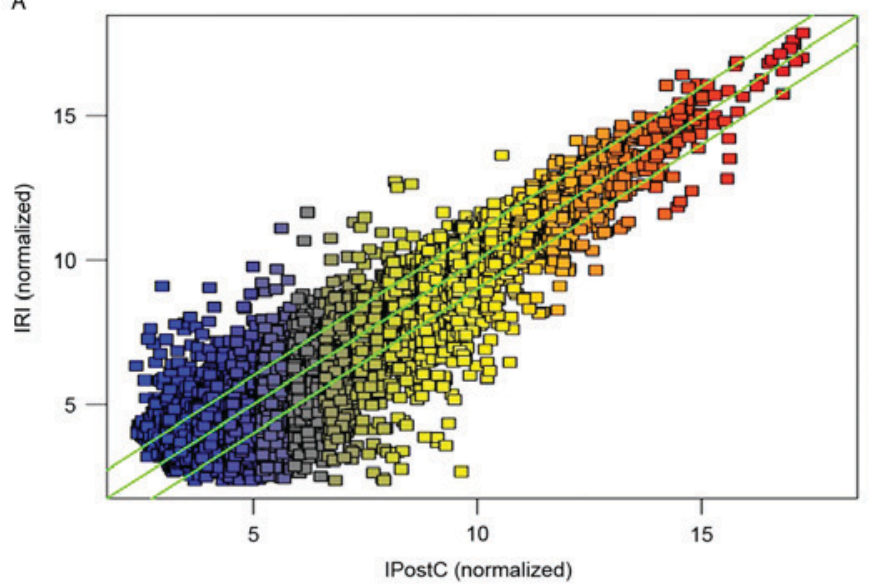

C

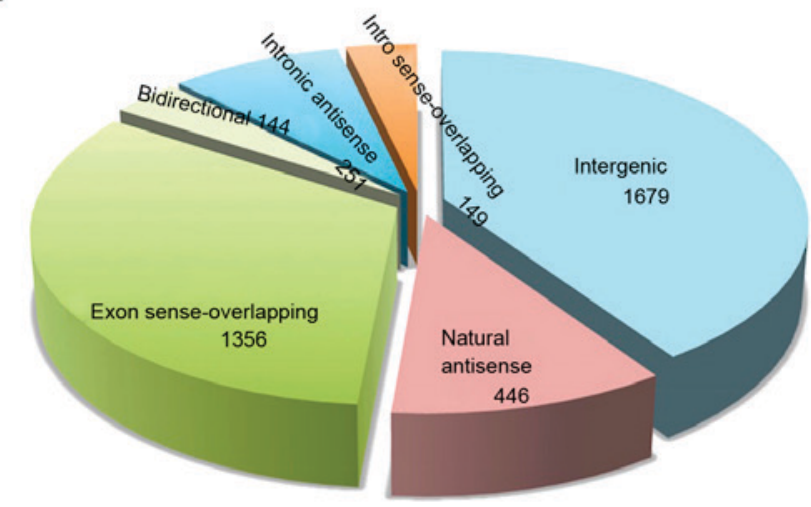

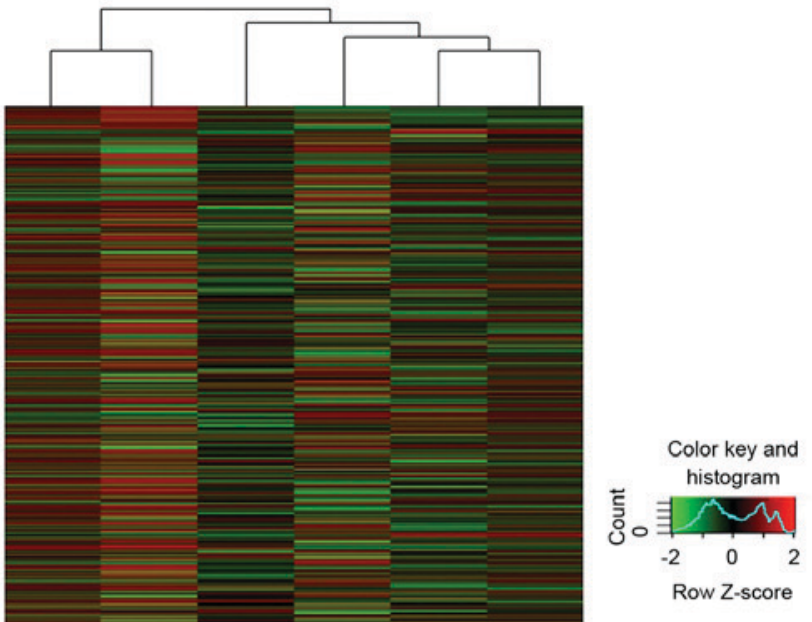

Figure 3. Differential lncRNA expression characters between IRI and IPostC tissues. (C) The lncRNA microarray revealed differences of lncRNA expression between IRI and IPostC via (A) hot-spot and (B) cluster mapping. Based on the locational association of the nearby coding genes, the differentially expressed lncRNAs were classified into various types, including 446 natural antisense, 361 intronic antisense, 149 intron sense-overlapping, 1,679 intergenic, 1,356 exon sense-overlapping and 144 bidirectional lncRNAs. IRI, ischemic reperfusion injury; IPostC, ischemic post-conditioning; lncRNAs, long non-coding RNAs.

of increasing interest to researchers. Numerous interventions have been tested in human trials continuing on from positive results of animal studies. IPostC refers to the transient ischemia/reperfusion cycle prior to reperfusion for cases of myocardial ischemia. Zhao et al (3) confirmed that IPostC improves ischemic perfusion in a canine acute ischemia and reperfusion model. IPostC exhibits an important practical application value and research regarding its effects is of primary concern in the cardiovascular field. At present, the exact protective effects of IPostC on myocardial reperfusion and the associated molecular mechanism remains to be elucidated.

The mortality rate of cardiovascular disease has not been decreased, and increasing efforts have been made to understand the link between heart disease and novel therapeutic targets, including non-coding RNAs. Of the multiple categories of non-coding RNAs, IncRNAs have emerged as novel therapeutics in cardiovascular medicine. LncRNAs are endogenous RNAs that contain $>200$ nucleotides and regulate gene expression. Various lncRNAs have recently been demonstrated to be important in regulating the physiological behavior of malignant cancers, including breast, pancreatic, gastric and lung. Notably, lncRNAs have primarily been revealed to regulate cancer cell viability, apoptosis, invasion and metastasis (18-24). Various dysregulated lncRNAs between IR and adjacent normal tissues have been identified (25), however, information and mechanisms regarding the differentially expressed lncRNAs between IR and IPostC tissue remains to be elucidated. The present study aimed to improve the understanding of the lncRNA expression character in IPostC.

The results of microarray assay revealed the dysregulated lncRNAs in IPostC compared with IR tissue (Fig. 3A and B), including 466 natural antisense and 1,679 intergenic (Fig. 3C). According to the locational association of the nearby coding genes, differentially expressed lncRNAs primarily included natural antisense, intronic antisense, intron sense-overlapping, intergenic, exon sense-overlapping and bidirectional lncRNAs. Previous studies have revealed that natural antisense and intergenic are more likely to regulate biological processes $(17,39)$. The data therefore suggested that the ameliorative effects of IPostC were associated with the differentially expressed lncRNAs. In order to predict the potential function of the dysregulated lncRNAs, GO analysis was conducted. The GO analyzed results indicated that these gene products were primarily found in the extracellular space, extracellular region, intracellular, intracellular part and intracellular organelle (Fig. 4A). The genes were enriched in the biological processes of immune system process, single-multicellular organism process and response to stimulus (Fig. 4B) which are associated to malignancy of the heart. The potential 

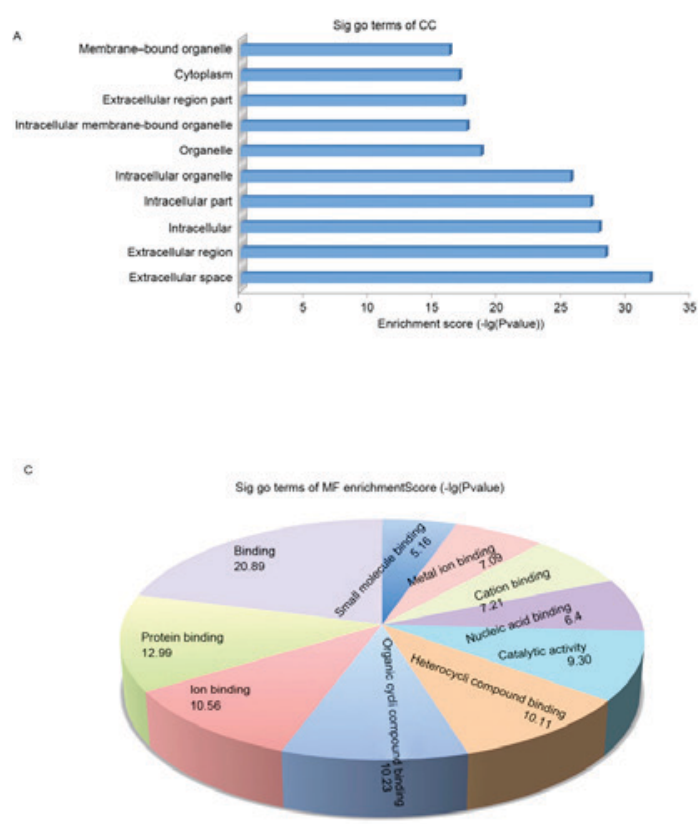
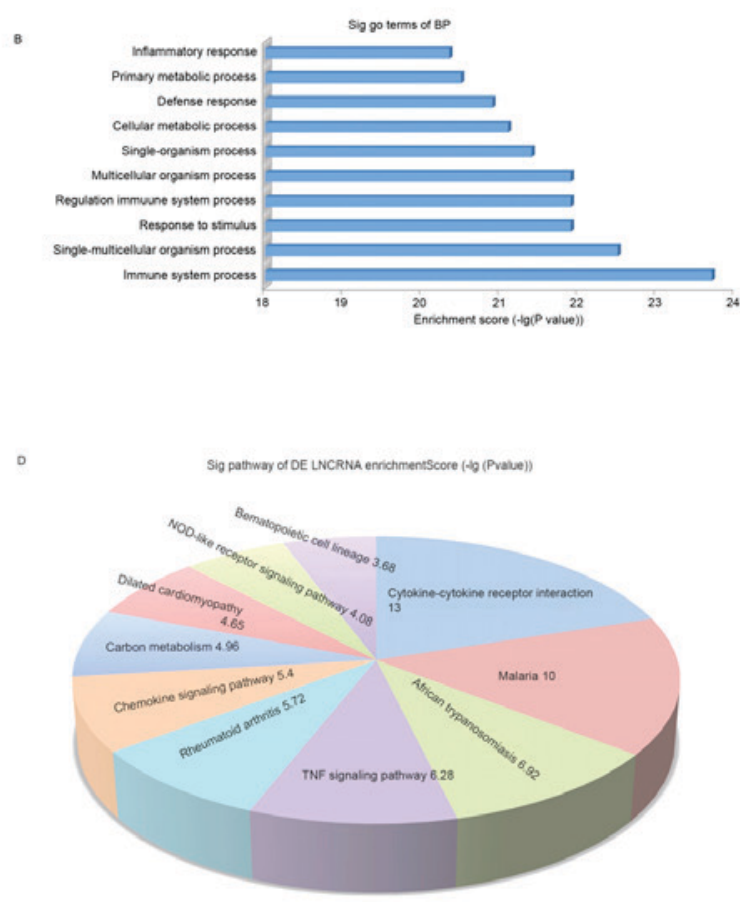

Figure 4. GO and Pathway analysis were performed to explore the potential functions of the dysregulated long non-coding RNAs in ischaemic post-conditioning. GO analysis data revealed that gene products were primarily located in (A) extracellular space, extracellular region, intracellular and intracellular parts. (B) The top 10 biological processes in which targeted genes participated. (C) The MF of these genes included binding, protein binding, organic cyclic compound binding and ion binding. The pathway analysis result indicated that these genes were involved in (D) cytokine-cytokine receptor interactions, malaria, African trypanosomiasis, tumor necrosis factor signaling, rheumatoid arthritis, chemokine signaling, carbon metabolism and dilated cardiomyopathy. MF, molecular function; GO, gene ontology.

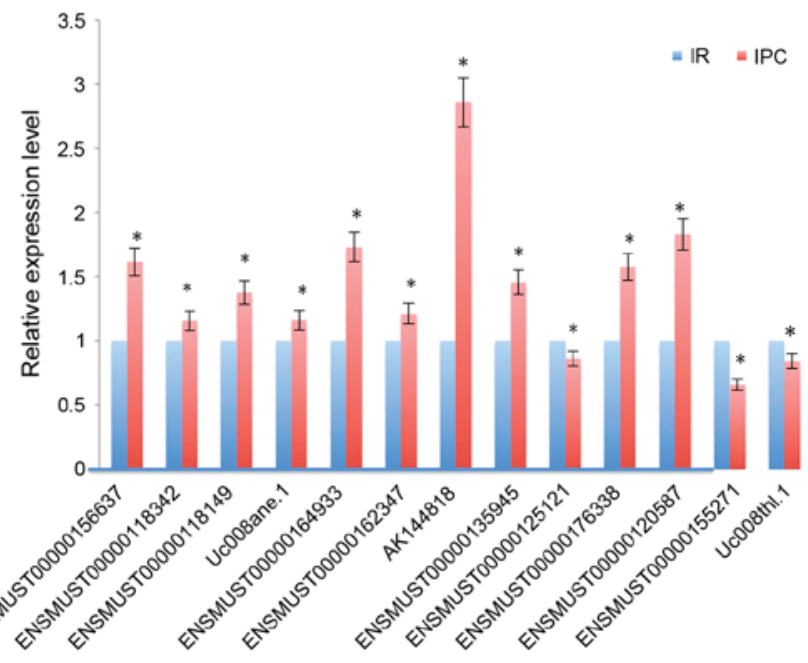

Figure 5. Validation of IPostC-associated lncRNAs. The expression levels of dysregulated lncRNAs in IRI and IpostC tissues, with 12 lncRNAs dysregulated in IpostC compared with IRI samples. Each experiment was repeated in triplicate. ${ }^{*} \mathrm{P}<0.05$ vs. IR. IRI, ischemic reperfusion injury. IR, ischemic reperfusion; IPC, IPostC, ischemic post-conditioning; lncRNAs, long non-coding RNAs.

functions were additionally classified into 10 categories via analysis of the target gene pool (Fig. 4C), involving binding, protein binding, ion binding, organic cycli compound binding, heterocycli compound binding, catalytic activity, nucleic acid binding, cation binding, metal ion binding, small molecule binding. Notably, it was observed that the dysregulated lncRNAs were able to be divided into two groups of binding and ligase activity, suggesting they may exhibit important roles in biological processes via regulation of the cell skeleton. Furthermore, pathway analysis results revealed that the dysregulated lncRNAs predominantly participated in signaling pathways (Fig. 4D), including cytokine-cytokine receptor interaction (mmu04060), malaria (mmu05144), African trypanosomiasis (mmu05143), tumor necrosis factor signaling pathway (mmu04668), rheumatoid arthritis (mmu05323), chemokine signaling pathway (mmu04062), carbon metabolism (mmu01200) and dilated cardiomyopathy (mmu05414). Dilated cardiomyopathy, arrhythmogenic right ventricular dysplasia, fanconi anemia and hypertrophic cardiomyopathy have been studied in the initiation and development of IR. It was observed that 500 dysregulated lncRNAs are involved in dilated cardiomyopathy (25). It remains unknown if an association is present between the development of dilated cardiomyopathy and IPostC. The differentially expressed lncRNAs partially indicated the molecular character in IPostC, compared with the IR tissues, and these lncRNAs may act as individual biomarkers for diagnosis, or therapeutic targets, for clinical IR treatment in the future.

The expression levels of the dysregulated lncRNAs were confirmed in the 3 IPostC samples and 3 IR samples. The differentially expressed IncRNAs were selected as described previously, and the RT-qPCR results demonstrated that compared with IR tissues, AK144818, ENS 
MUST00000156637, ENSMUST00000118342, ENSMUS T00000118149, Uc008ane.1, ENSMUST00000164933, ENS MUST00000162347, ENSMUST00000135945, ENSMUS T00000176338 and ENSMUST00000120587 were markedly upregulated in IPostC tissues (Fig. 5). Conversely, ENSMUS T00000125121, ENSMUST00000155271 and Uc008thl.1 were downregulated. ENSMUST00000176338 is a lncRNA which targets the Gm20621 gene, ENSMUST00000156276 is an exon sense-overlapping IncRNA which targets Cd209f, whereas all of the 8 upregulated are intergenic lncRNAs.

It has previously been demonstrated that intergenic and antisense lncRNAs regulate cell activity in various types of cancer (40-44), suggesting this as a potential function of the 8 upregulated lncRNAs in IPostC. LncRNAs may additionally act as endogenous inhibitors to reverse the effects of miRNAs $(45,46)$.

In conclusion, dysregulated lncRNAs may therefore act as novel biomarkers for diagnosis of IRI and potential targets for the pharmacogenetic therapy of cardiovascular patients in the future.

\section{Acknowledgements}

The present study was supported by the National Natural Science Foundation of China (grant nos. 81173052, 30973534 and 81402139). The authors would like to thank KangChen Bio-tech Ltd. (Shanghai, China), and in particular, Dr Feifei $\mathrm{Xu}$ and $\mathrm{Mr}$ Yajie Zhang for the IncRNA microarray analysis.

\section{References}

1. Writing Group Members, Lloyd-Jones D, Adams RJ, Brown TM, Carnethon M, Dai S, De Simone G, Ferguson TB, Ford E, Furie K, et al: Heart disease and stroke statistics-2010 update: A report from the American heart association. Circulation 121: e46-e215, 2010.

2. Hearse DJ: Reperfusion of the ischemic myocardium. J Mol Cell Cardiol 9: 605-616, 1977

3. Zhao ZQ, Corvera JS, Halkos ME, Kerendi F, Wang NP, Guyton RA and Vinten-Johansen J: Inhibition of myocardial injury by ischemic postconditioning during reperfusion: Comparison with ischemic preconditioning. Am J Physiol Heart Circ Physiol 285: H579-H588, 2003.

4. Przyklenk K, Bauer B, Ovize M, Kloner RA and Whittaker P: Regional ischemic 'preconditioning' protects remote virgin myocardium from subsequent sustained coronary occlusion. Circulation 87: 893-899, 1993.

5. Hausenloy DJ and Yellon DM: Remote ischaemic preconditioning: Underlying mechanisms and clinical application. Cardiovasc Res 79: 377-386, 2008.

6. O'Neill WW, Martin JL, Dixon SR, Bartorelli AL, Trabattoni D, Oemrawsingh PV, Atsma DE, Chang M, Marquardt W, Oh JK, et al: Acute myocardial infarction with hyperoxemic therapy (AMIHOT): A prospective, randomized trial of intracoronary hyperoxemic reperfusion after percutaneous coronary intervention. J Am Coll Cardiol 50: 397-405, 2007.

7. Duncker DJ, Klassen CL, Ishibashi Y, Herrlinger SH, Pavek TJ and Bache RJ: Effect of temperature on myocardial infarction in swine. Am J Physiol 270: H1189-H1199, 1996.

8. Quindry JC, Miller L, McGinnis G, Kliszczewicz B, Irwin JM, Landram M, Urbiztondo Z, Nanayakkara G and Amin R: Ischemia reperfusion injury, KATP channels, and exercise-induced cardioprotection against apoptosis. J Appl Physiol (1985) 113: 498-506, 2012.

9. Ruiz-Meana M, Inserte J, Fernandez-Sanz C, Hernando V, Miro-Casas E, Barba I and Garcia-Dorado D: The role of mitochondrial permeability transition in reperfusion-induced cardiomyocyte death depends on the duration of ischemia. Basic Res Cardiol 106: 1259-1268, 2011.
10. Zheng $\mathrm{X}, \mathrm{Zu} \mathrm{L}$, Becker L and Cai ZP: Ischemic preconditioning inhibits mitochondrial permeability transition pore opening through the PTEN/PDE4 signaling pathway. Cardiology 129: 163-173, 2014.

11. Hu L, Zhou L, Wu X, Liu C, Fan Y and Li Q: Hypoxic preconditioning protects cardiomyocytes against hypoxia/reoxygenation injury through AMPK/eNOS/PGC-1 $\alpha$ signaling pathway. Int J Clin Exp Pathol 7: 7378-7388, 2014.

12. Raedschelders K, Ansley DM and Chen DD: The cellular and molecular origin of reactive oxygen species generation during myocardial ischemia and reperfusion. Pharmacol Ther 133: 230-255, 2012.

13. Murry CE, Jennings RB and Reimer KA: Preconditioning with ischemia: A delay of lethal cell injury in ischemic myocardium. Circulation 74: 1124-1136, 1986.

14. Rinn JL and Chang HY: Genome regulation by long noncoding RNAs. Annu Rev Biochem 81: 145-166, 2012.

15. Latos PA, Pauler FM, Koerner MV, Şenergin HB, Hudson QJ, Stocsits RR, Allhoff W, Stricker SH, Klement RM, Warczok KE, et al: Airn transcriptional overlap, but not its lncRNA products, induces imprinted Igf2r silencing. Science 338: 1469-1472, 2012.

16. Khalil AM, Guttman M, Huarte M, Garber M,Raj A, Rivea Morales D, Thomas K, Presser A, Bernstein BE, van Oudenaarden A, et al: Many human large intergenic noncoding RNAs associate with chromatin-modifying complexes and affect gene expression. Proc Natl Acad Sci USA 106: 11667-11672, 2009.

17. Ponting CP, Oliver PL and Reik W: Evolution and functions of long noncoding RNAs. Cell 136: 629-641, 2009.

18. Li H, Yu B, Li J, Su L, Yan M, Zhu Z and Liu B: Overexpression of lncRNA H19 enhances carcinogenesis and metastasis of gastric cancer. Oncotarget 5: 2318-2329, 2014.

19. Jin G, Sun J, Isaacs SD, Wiley KE, Kim ST, Chu LW, Zhang Z, Zhao H, Zheng SL, Isaacs WB and Xu J: Human polymorphisms at long non-coding RNAs (lncRNAs) and association with prostate cancer risk. Carcinogenesis 32: 1655-1659, 2011.

20. Arase M, Horiguchi K, Ehata S, Morikawa M, Tsutsumi S, Aburatani H, Miyazono K and Koinuma D: Transforming growth factor- $\beta$-induced lncRNA-Smad7 inhibits apoptosis of mouse breast cancer JygMC(A) cells. Cancer Sci 105: 974-982, 2014.

21. Wang K, Long B, Zhou LY, Liu F, Zhou QY, Liu CY, Fan YY and Li PF: CARL lncRNA inhibits anoxia-induced mitochondrial fission and apoptosis in cardiomyocytes by impairing miR-539-dependent PHB2 downregulation. Nat Commun 5: 3596, 2014.

22. Yang L, Lin C, Jin C, Yang JC, Tanasa B, Li W, Merkurjev D, Ohgi KA, Meng D, Zhang J, et al: lncRNA-dependent mechanisms of androgen-receptor-regulated gene activation programs. Nature 500: 598-602, 2013.

23. Wang K, Liu CY, Zhou LY, Wang JX, Wang M, Zhao B, Zhao WK, Xu SJ, Fan LH, Zhang XJ, et al: APF lncRNA regulates autophagy and myocardial infarction by targeting miR-188-3p. Nat Commun 6: 6779, 2015.

24. Shi SJ, Wang LJ, Yu B, Li YH, Jin Y and Bai XZ: lncRNA-ATB promotes trastuzumab resistance and invasion-metastasis cascade in breast cancer. Oncotarget 6: 11652-11663, 2015.

25. Archer K, Broskova Z, Bayoumi AS, Teoh JP, Davila A, Tang Y, $\mathrm{Su} \mathrm{H}$ and Kim IM: Long non-coding RNAs as master regulators in cardiovascular diseases. Int J Mol Sci 16: 23651-23667, 2015.

26. Zu L, Zheng X, Wang B, Parajuli N, Steenbergen C, Becker LC and Cai ZP: Ischemic preconditioning attenuates mitochondrial localization of PTEN induced by ischemia-reperfusion. Am J Physiol Heart Circ Physiol 300: H2177-H2186, 2011.

27. Hu L, Wang J, Zhu H, Wu X, Zhou L, Song Y, Zhu S, Hao M, Liu C, Fan Y, et al: Ischemic postconditioning protects the heart against ischemia-reperfusion injury via neuronal nitric oxide synthase in the sarcoplasmic reticulum and mitochondria. Cell Death Dis 7: e2222, 2016

28. Suzuki M, Saito T, Sato T, Tamagawa M, Miki T, Seino S and Nakaya H: Cardioprotective effect of diazoxide is mediated by activation of sarcolemmal but not mitochondrial ATP-sensitive potassium channels in mice. Circulation 107: 682-685, 2003.

29. Lv M, Xu P, Wu Y, Huang L, Li W, Lv S, Wu X, Zeng X, Shen R, Jia X, et al: lncRNAs as new biomarkers to differentiate triple negative breast cancer from non-triple negative breast cancer. Oncotarget 7: 13047-13059, 2016.

30. Livak KJ and Schmittgen TD: Analysis of relative gene expression data using real-time quantitative PCR and the 2(-Delta Delta C(T)) method. Methods 25: 402-408, 2001. 
31. Spurlock CF III, Tossberg JT, Guo Y, Collier SP, Crooke PS III and Aune TM: Expression and functions of long noncoding RNAs during human Thelper cell differentiation. Nat Commun 6: 6932, 2015.

32. Balakrishnan R, Harris MA, Huntley R, Van Auken K and Cherry JM: A guide to best practices for Gene Ontology (GO) manual annotation. Database (Oxford) 2013: bat054, 2013.

33. Khatri P, Sirota M and Butte AJ: Ten years of pathway analysis: Current approaches and outstanding challenges. PLoS Comput Biol 8: e1002375, 2012.

34. Mäkinen N, Vahteristo P, Kämpjärvi K, Arola J, Bützow R and Aaltonen LA: MED12 exon 2 mutations in histopathological uterine leiomyoma variants. Eur J Hum Genet 21: 1300-1303, 2013.

35. Cook JD and Walker CL: The Eker rat: Establishing a genetic paradigm linking renal cell carcinoma and uterine leiomyoma. Curr Mol Med 4: 813-824, 2004.

36. Ptacek T, Song C, Walker CL and Sell SM: Physical mapping of distinct $7 \mathrm{q} 22$ deletions in uterine leiomyoma and analysis of a recently annotated 7q22 candidate gene. Cancer Genet Cytogenet 174: 116-120, 2007.

37. Mattick JS: The central role of RNA in human development and cognition. FEBS Lett 585: 1600-1616, 2011.

38. Wapinski $\mathrm{O}$ and Chang HY: Long noncoding RNAs and human disease. Trends Cell Biol 21: 354-361, 2011

39. Necsulea A, Soumillon M, Warnefors M, Liechti A, Daish T, Zeller U, Baker JC, Grützner F and Kaessmann H: The evolution of lncRNA repertoires and expression patterns in tetrapods. Nature 505: 635-640, 2014

40. Cui W, Qian Y, Zhou X, Lin Y, Jiang J, Chen J, Zhao Z and Shen B: Discovery and characterization of long intergenic non-coding RNAs (lincRNA) module biomarkers in prostate cancer: An integrative analysis of RNA-Seq data. BMC Genomics 16 (Suppl 7): S3, 2015.
41. Hu Y, Wang J, Qian J, Kong X, Tang J, Wang Y, Chen H, Hong J, Zou W, Chen Y, et al: Long noncoding RNA GAPLINC regulates CD44-dependent cell invasiveness and associates with poor prognosis of gastric cancer. Cancer Res 74: 6890-6902, 2014.

42. Wu Y, Liu H, Shi X, Yao Y, Yang W and Song Y: The long non-coding RNA HNF1A-AS1 regulates proliferation and metastasis in lung adenocarcinoma. Oncotarget 6: 9160-9172, 2015.

43. Lu L, Zhu G, Zhang C, Deng Q, Katsaros D, Mayne ST, Risch HA, Mu L, Canuto EM, Gregori G, et al: Association of large noncoding RNA HOTAIR expression and its downstream intergenic $\mathrm{CpG}$ island methylation with survival in breast cancer. Breast Cancer Res Treat 136: 875-883, 2012.

44. Kim K, Jutooru I, Chadalapaka G, Johnson G, Frank J, Burghardt R, Kim S and Safe S: HOTAIR is a negative prognostic factor and exhibits pro-oncogenic activity in pancreatic cancer. Oncogene 32: 1616-1625, 2013.

45. Liang WC, Fu WM, Wong CW, Wang Y, Wang WM, Hu GX, Zhang L, Xiao LJ, Wan DC, Zhang JF and Waye MM: The lncRNA H19 promotes epithelial to mesenchymal transition by functioning as miRNA sponges in colorectal cancer. Oncotarget 6: 22513-22525, 2015.

46. Cai H, Xue Y, Wang P, Wang Z, Li Z, Hu Y, Li Z, Shang X and Liu Y: The long noncoding RNA TUG1 regulates blood-tumor barrier permeability by targeting miR-144. Oncotarget 6 : 19759-19779, 2015. 